\title{
Large scale chirality transduction with functional molecular materials
}

\author{
Serena Arnaboldi $i^{1,3}$, Bhavana Gupta ${ }^{1,5 \ddagger}$, Tiziana Benincori ${ }^{2}$, Giorgia Bonetti ${ }^{2}$, Roberto Cirilli ${ }^{4}$, \\ Alexander Kuhn ${ }^{1 *}$ \\ ${ }^{1}$ Univ. Bordeaux, ISM CNRS UMR 5255, Bordeaux INP, ENSCBP, 16 avenue Pey Berland, 33607 Pessac, France, \\ *kuhn@enscbp.fr \\ ${ }^{2}$ Univ.degli Studi dell'Insubria, Dip. di Scienza e Alta Tecnologia, Via Valleggio 11, 22100 Como, Italy \\ ${ }^{3}$ Univ. degli Studi di Milano, Dip. di Chimica, Via Golgi 19, 20133 Milano; Italy \\ ${ }^{4}$ Centro Nazionale per il Controllo e la Valutazione dei Farmaci, Istituto Superiore di Sanità, Viale Regina Elena 299, 00161 \\ Roma, Italy \\ ${ }^{5}$ National \& Local Joint Engineering Research Center for Applied Technology of Hybrid Nanomaterials, Henan University, \\ Kaifeng 475004, China.
}

\begin{abstract}
Transduction of chiral information can be achieved at different length scales. Among all possible approaches, we propose in this work a straightforward concept to transfer chiral features from the molecular level to the shape of macroscopic objects by combining the concepts of inherently chiral oligomers and bipolar electrochemistry. Hybrid freestanding lamellar films, composed of polypyrrole and the two oligomeric antipodes of a chiral monomer, are exposed in solution to a chiral target molecule (i.e. L- and D-DOPA) in the presence of an electric field. This leads to an electrochemically induced deformation of the film, which in fine results in one or the other of two macroscopic enantiomorphs, depending on which stereoisomer is present in the solution.
\end{abstract}

\section{INTRODUCTION}

Chirality can be defined at different length scales, ranging from the molecular to the supramolecular and macroscopic scale $^{1}$. Local chirality refers to the molecular scale, and arises from variations in the configuration of a molecule. Global chirality is defined with respect to conformations at bigger length scales, such as in oligomers, polymers and supramolecular assemblies. Macroscopic chirality refers to micrometer or larger scales, including crystals and biological structures.

The transmission mechanism of chirality from nano- to meso- and macroscopic length scales is rather complex and not yet well understood ${ }^{1}$. Since the first resolution experiments carried out by Pasteur in 1848, many studies have addressed this fascinating topic of chirality transfer from the molecular scale to structures at the macroscopic level ${ }^{2-4}$. Various biological materials, such as plant tendrils, flower petals and snail shells, exhibit chiral growth. In these natural materials, there are chiral elements that lead to the formation of various chiral morphologies at the macroscopic scale with a specific handedness, either right or left. The investigation of the mechanisms related to chiral growth of biological materials is a fundamental issue, not only in biology, but also closely connected to materials science and technology. Scientists have explored chirality transmission mechanisms at different length scales with several concepts that in general try to mimic what happens in nature.

The most obvious way to transfer chirality across scales is through chiral recognition and/or enantioselective interactions between molecules ${ }^{5}$. In chemistry, these processes are usually studied by optical, spectroscopic and electrochemical methods. The transduction of a molecular chiral feature into macroscopic changes of the properties of materials is extremely important and directly connected to practical applications like chiral separation, catalysis and functional devices ${ }^{6-7}$.

Among the artificial systems which mimic natural ones, one can cite chiral liquid crystals $^{8}$, chiral metamaterials ${ }^{9}$, chiral catalysts $^{10}$, chiral biosensors ${ }^{11}$, and chiral separation materials $^{12}$.

In this context electronically conducting polymers are particularly interesting materials which can be employed in advanced devices, due to their good electronic conductivity and the possibility to easily tune their properties by functionalisation. ${ }^{13}$ These materials are able to act concurrently as receptors and transducers, and are either directly endowed with chirality, or a chiral molecule can be integrated during polymerization. In this way, chiral information can be transduced from the chiral guest molecules, or the polymer itself, into macroscopic features of the material. On the other hand, the properties of conducting polymers are also modified by external stimuli (solvent ${ }^{14-15}$, temperature ${ }^{16}, \mathrm{pH}^{16}$, electric field $^{17}$ ) that may directly impact the chiral conformation of the polymer chains at the macromolecular level.

Enantioselective interactions between chiral molecules and chiral conducting polymers can induce visible changes at the macroscopic scale. A variety of polymers/oligomers have been developed so far, mostly including chiral thiophene, pyrrole and aniline derivatives. Some of these materials have been 
tested for enantioselection discrimination in advanced electrochemical sensing devices. ${ }^{18}$ However, in many cases the enantioselectivity is rather weak and the response (e.g., in terms of current or potential $v s$ concentration) seems unsuitable for the recognition and quantification of both enantiomeric configurations of a probe. This is due to the fact that often the stereogenic elements are either external to the main backbone $^{19}$ or related to a secondary chiral structure which can be easily lost during operation. ${ }^{20}$ The same is true for chiral features which originate from external templating agents. ${ }^{21}$

In 2010 Sannicolò et al. designed a monomer ${ }^{22}$ with 3,3'bibenzothiophene as a central scaffold, endowed with inherent chirality, ${ }^{23}$ to be employed as racemic co-monomer in electrosynthesis. In other reports, tests were carried out using the individual enantiomers of this chiral molecule, separated by chiral HPLC. ${ }^{24}$ Their electrooligomerization resulted in chiral electrode surfaces, able to discriminate the enantiomers of chiral species by differences in peak potential recorded by cyclic voltammetry. ${ }^{25-26}$ This is a true advantage from an analytical point of view, because it is possible to achieve discrimination of racemic mixtures of the analyte, in contrast to most of the approaches discussed above for which the recognition is only based on differences in current intensity ${ }^{27-29}$.

In this context, a new concept of absolute chiral recognition has been reported very recently ${ }^{30}$, based on the chirality dependent induction of motion of a hybrid polymer film. Analog to previous works ${ }^{31-32}$, bipolar electrochemistry was used as a driving force to trigger the preferential oxidative conversion of an enantiomer at one extremity of the polymer film, whereas at the opposite extremity the polymer undergoes a reduction, accompanied by site selective swelling and shrinking. The resulting deformation of the film can then be used as a readout of chiral information. In contrast to previous studies ${ }^{33-34}$, the combination of the robust concept of inherent chirality with the electromechanical proprieties of polypyrrole (Ppy), allowed the development of a bipolar electroactuator with a true chirality-dependent on-off behavior ${ }^{30}$. The enantiopure inherently chiral oligomer was deposited on one side of a Ppy strip and used as bipolar electrode able to react differently, with a yes-no response, to the enantiomers of a redox active chiral analyte (L- or D-DOPA). The difference in terms of oxidation potentials between L- and D-DOPA is sufficient to observe the electromechanical actuation of Ppy exclusively in the presence of the enantiomer that is easier to oxidize. Furthermore, the actuation is proportional to the concentration of the "good" enantiomer, and completely independent of the presence of the other antipode ${ }^{30}$.

In the present work, we intend to make a further step towards the transfer of chiral information from the molecular to the macroscopic scale. In particular, the aim is to trigger by bipolar electrochemistry the deformation of a specifically designed hybrid material in such a way that it leads to the formation of two macroscopic objects, being not superimposable mirror images of each other.

\section{RESULTS AND DISCUSSION}

The electrooligomerization of enantiopure inherently chiral monomers on substrates like gold or glassy carbon in different media (aqueous media and organic solvents) leads to enantiopure surfaces with a well-pronounced enantioselection ability ${ }^{18-21}$. In order to confirm in a first step that the oligomers show chiral recognition properties also when they are generat- ed on a Ppy film, we carried out tests with Ppy films, modified with $(R)$ or $(S)$ enantiopure oligomer layers, analog to what has been reported earlier ${ }^{25}$. The inherently chiral monomer chosen for this purpose was 2,2'-bis[2-(5,2'-bithienyl)]-3,3'bithianaphthene $\left(\mathrm{BT}_{2} \mathrm{~T}_{4}\right)$, already well-known and characterized by Mussini et al. ${ }^{18-21}$. It is constituted of 3,3'bibenzothiophene as an atropisomeric central scaffold, with a sufficiently high racemization barrier to yield stable enantiomers, and functionalized with two 2,2'-bithiophenic units, featuring two $\alpha$-homotopic positions of the terminal thiophene rings, granting a regioregular oligomerization. In preliminary tests, Ppy films were deposited by chronopotentiometry on gold coated glass slides and then covered with oligo- $(R)$ or oligo- $(S)-\mathrm{BT}_{2} \mathrm{~T}_{4}$ according to a literature procedure ${ }^{30-31}$. The final hybrid films were peeled off from the gold-coated glass slide and used for a first, more classic enantiorecognition tests, performed by differential pulse voltammetry (DPV) in the presence of aqueous solutions of $5 \mathrm{mM} \mathrm{L}$ - or D-DOPA and lithium perchlorate $0.1 \mathrm{M}$.
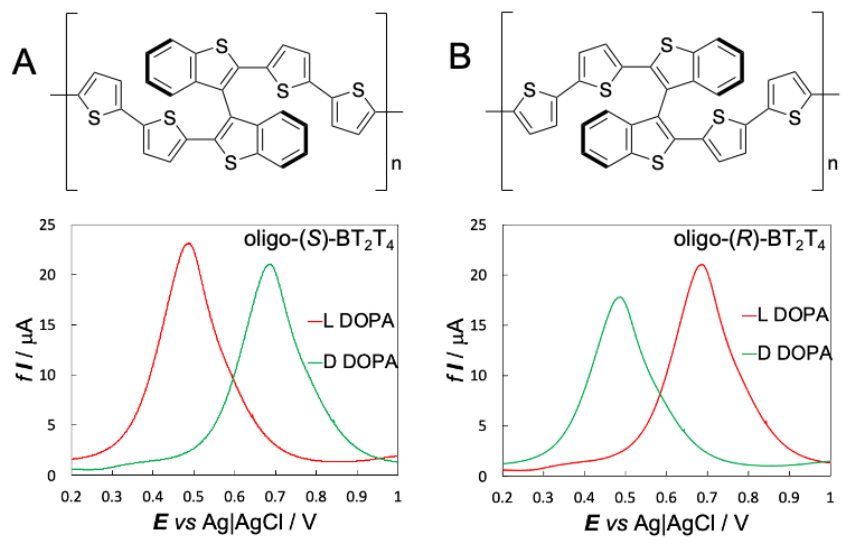

Figure 1. Top: Chemical structures of the enantiopure oligo- $(S)$ $\mathrm{BT}_{2} \mathrm{~T}_{4}(\mathrm{~A})$ and oligo- $(R)-\mathrm{BT}_{2} \mathrm{~T}_{4}(\mathrm{~B})$ molecules employed in the enantiorecognition tests. Bottom: Differential pulse voltammetry signals of the enantioselective electrooxidation of $5 \mathrm{mM} \mathrm{L}$ - or DDOPA in water and $0.1 \mathrm{M} \mathrm{LiClO}_{4}$ on a hybrid polymer layer composed of $\mathrm{A}) \mathrm{Ppy}+$ oligo- $(S)-\mathrm{BT}_{2} \mathrm{~T}_{4}$ and $\left.\mathrm{B}\right) \mathrm{Ppy}+$ oligo- $(R)-$ $\mathrm{BT}_{2} \mathrm{~T}_{4}$.

From Figure 1A, it is obvious that the $(S)$-hybrid surface is preferentially reacting with L-DOPA, with a peak-to-peak separation between the two enantiomers of about $200 \mathrm{mV}$. The results are also perfectly coherent when the opposite configuration of the $\mathrm{BT}_{2} \mathrm{~T}_{4}$ oligomer is used. The $(R)$-hybrid allows a preferential oxidation of D-DOPA. Consequently, at $\sim 0.45 \mathrm{~V}$ vs. $\mathrm{AgCl} / \mathrm{Ag}$ it is possible to selectively electrooxidize exclusively one of the two DOPA enantiomers, depending on which oligomer configuration is deposited on Ppy.

If this hybrid material is used as an electrode in a bipolar electrochemistry experiment, it should be possible to transform exclusively L- or D-DOPA on the positively polarized extremity of the film if the polarization potential difference is fine-tuned to a value that is just enough to oxidize the "good" enantiomer. At the other extremity of the bipolar object, a charge compensating reduction reaction has to occur simultaneously. Considering that Ppy reduction occurs at about -0.25 $\mathrm{V}$ vs. $\mathrm{AgCl} / \mathrm{Ag}$, the theoretical threshold of the polarization potential difference between the two extremities of the bipolar 
electrode should be around $0.65 \mathrm{~V}$ in order to activate both reactions at the same time.

The schematic cell configuration employed in the bipolar experiments is represented in Scheme 1A. Two graphite rods are used as feeder electrodes and connected to a power supply. The bipolar electrode has a specific shape and composition in order to allow transmission of chiral information from the molecular to the macroscopic scale. Actually, the overall geometry has to be a scalene triangle with its two faces being constituted of $(R)$ - and $(S)$-oligo-B $\mathrm{BT}_{2} \mathrm{~T}_{4}$, electrodeposited on Ppy, respectively. They are combined in a sandwich-type structure with an insulator in between in order to prevent electrons from passing from one face to the other (Scheme $1 \mathrm{~B}$ and C). The final triangular object is placed directly at the bottom of the cell without using any kind of support. The solution contains L- or D-DOPA ( $5 \mathrm{mM}$ ) dissolved in water and $0.1 \mathrm{M}$ $\mathrm{LiClO}_{4}$. When a suitable electric field is applied, only one of the two enantiopure oligomer faces is able to react as a function of which analyte enantiomer is present in the solution.
A

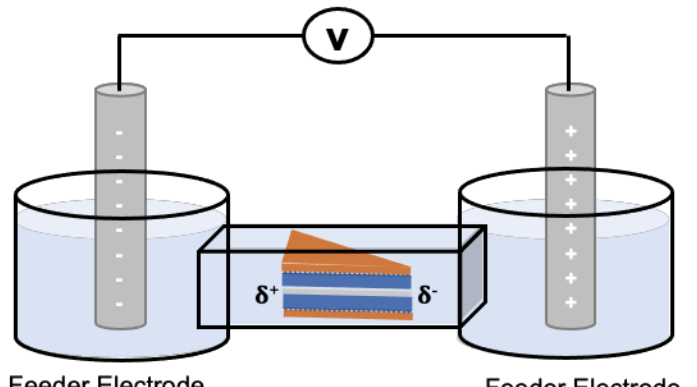

Feeder Electrode

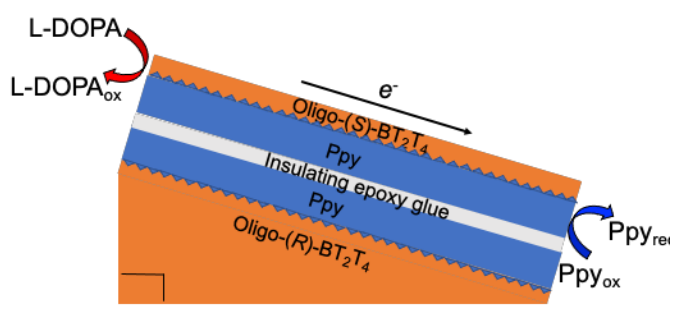

B

C

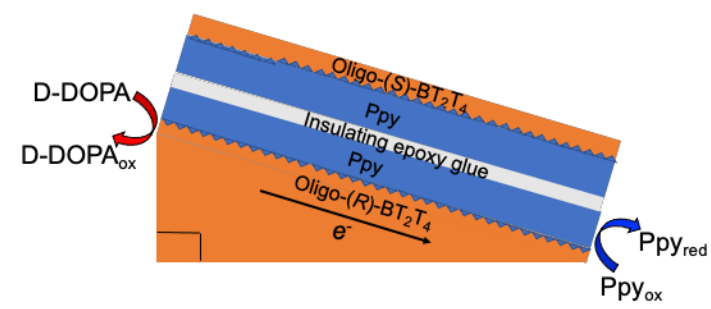

Scheme 1. A) Schematic illustration of the bipolar cell used for the experiments of chirality transmission through space. The distance between the feeder electrodes is $5 \mathrm{~cm}$ and the length of the bipolar electrode is $1.0 \mathrm{~cm}$. The solution contains $5 \mathrm{mM} \mathrm{L}$ - or D-DOPA in water and $0.1 \mathrm{M} \mathrm{LiClO}_{4}$. B) and C) Magnifications of the sandwich-like bipolar electrodes used for the experiments and the concept responsible for their operation. When L-DOPA is present in solution, only the oligo- $(S)-\mathrm{BT}_{2} \mathrm{~T}_{4}$ layer is able to react (B) and vice versa when D-DOPA is the analyte (C).

An electric field of proper amplitude leads to a $\delta^{+}$and $\delta^{-}$polarization at the extremities of the triangle. Under these conditions, one of the antipodes of DOPA gets selectively electrooxidized at the positively polarized end, while at the $\delta^{-}$ side Ppy is reduced. The latter is accompanied by an uptake of solvated $\mathrm{Li}^{+}$cations that enter the polymer film in order to maintain electroneutrality. ${ }^{35-37}$ Charge compensation could in theory also occur via a release of anions, however the dodecylbenzene sulfonate anions, present inside the polymer, are rather bulky and therefore remain in the polymer. This process induces a face-selective swelling of the chiral object at the negatively polarized extremity, depending on the stereochemistry of both the chiral analyte in solution and the chiral oligomer.

The result of this asymmetric electromechanical bending is that the hybrid polymer film starts curling in one direction or the other. As the initial geometry is a scalene triangle, meaning that no sides and no angles are equal, the final curved objects have no plane of symmetry and thus are macroscopic enantiomorphs as illustrated in the following.

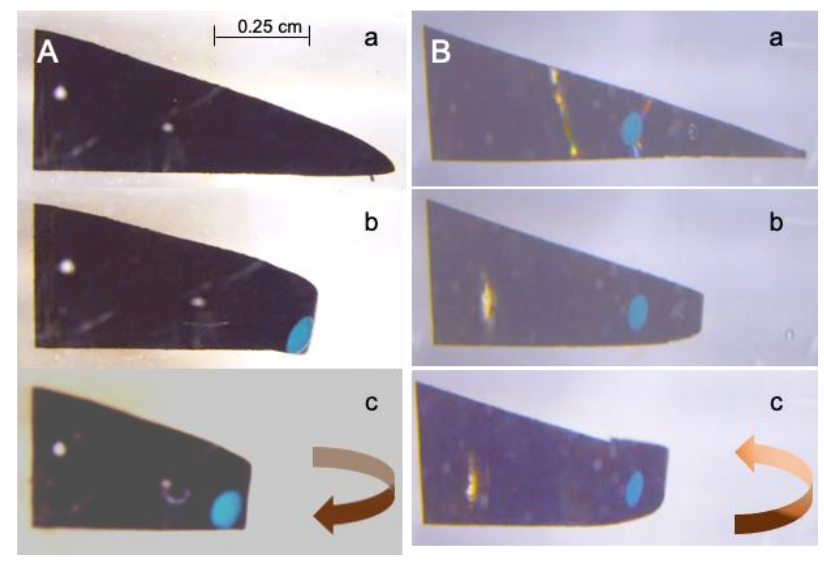

Figure 2. Hybrid lamellar polymer strips exposed to an aqueous solution of $5 \mathrm{mM}$ D-DOPA and $0.1 \mathrm{M} \mathrm{LiCO}_{4}$. A) $(R)$-oligo- $\mathrm{BT}_{2} \mathrm{~T}_{4}$ is oriented towards the backside. $\mathrm{B})(R)$-oligo- $\mathrm{BT}_{2} \mathrm{~T}_{4}$ is present at the front side. (a), (b) and (c) illustrate different stages during the bending process. A blue varnish dot is placed at the face modified with $(R)$-oligo- $\mathrm{BT}_{2} \mathrm{~T}_{4}$ to guide the eye during the movement induced by the application of an electric field of $0.6 \mathrm{~V} \mathrm{~cm}^{-1}$.

The system was optimized considering that the total hybrid film should not be too thick in order to guarantee $i$ ) electroactivity throughout the entire Ppy film, ii) an easy diffusion of $\mathrm{Li}^{+}$ions into the PPy matrix upon its reduction and iii) sufficient flexibility that allows the object's deformation by the electric field. A total thickness of $240 \mu \mathrm{m}$ (see Figure $\mathrm{S} 1$ ) is a good compromise in order to satisfy the different requirements. The deposition of Ppy and its thickness were already optimized and discussed in previous works ${ }^{30-32}$. The insulating layer, which has a thickness of around $100 \mu \mathrm{m}$, is as thin as technically possible in order to avoid additional stiffness. The capping enantiopure oligomer layer is very thin (less than $1 \mu \mathrm{m}$, Figure S2) as it only serves as molecular recognition element, but is not actively involved in the bending.

Placing the hybrid object in the bipolar cell in the presence of L- or D-DOPA and applying an electric field of $0.6 \mathrm{~V} \mathrm{~cm}^{-1}$, the direction of curling is controlled by the chiral face involved in the electrooxidation, as illustrated in Figure 2 (see also Video S1 and S2). A blue spot of varnish has been added in order to better visualize the curling and it is positioned at the face modified with $(R)$-oligo- $\mathrm{BT}_{2} \mathrm{~T}_{4}$ to guide the eye. Both chiral hybrid films were exposed to a solution containing 5 $\mathrm{mM}$ D-DOPA and $0.1 \mathrm{M} \mathrm{LiClO}_{4}$. In Figure 2A, the $(R)$-oligo$\mathrm{BT}_{2} \mathrm{~T}_{4}$ is present at the back side (blue spot not visible in (a)), whereas in Figure 2B the same oligomer was deposited on the front side of the hybrid object. In both cases, electrooxidation 
of D-DOPA occurs on the face modified with the $(R)$ oligomer, causing a swelling of this part of the bilamellar strip. In fine this results in a controlled bending of the film, either towards the front (Figure 2A b-c, the blue spot which initially was not visible moves towards the front) or backwards (Figure 2B b-c, the initially visible blue spot moves towards the backside).

Both experiments can also be followed in real time by watching the bending of the hybrid sheets from the side (Figure 3). In this case, the blue spot of varnish has always been added to the upper part of the lamellar object. In Figure $3 \mathrm{~A}$ the $(R)-\mathrm{BT}_{2} \mathrm{~T}_{4}$ oligomer is localized at the bottom face of the sheet in both cases ((a) and (b)). If these two identical objects are exposed to the molecular antipodes of DOPA, the bending occurs in opposite directions. In case (a), the bottom layer of oligomer reacts with the D-DOPA present in solution, because at the chosen polarization potential only this one can react with D-DOPA (see CVs in Figure 1 and Supporting Video S3). This provides the electrons necessary for the reduction of Ppy. Consequently the bottom Ppy layer swells and thus starts bending upwards. For case (b), with L-DOPA in solution, it is the upper layer of the hybrid film, composed of $(S)$-oligo$\mathrm{BT}_{2} \mathrm{~T}_{4}$ and Ppy, which becomes active. Therefore, the upper part starts swelling and the film is bending downwards. A perfectly complementary scenario of chiral selection is illustrated in Figure 3B. In this example the same enantiomer (DDOPA) is present in both experiments (a) and (b), however the hybrid layer has a different initial orientation. In (a) the $(R)$ $\mathrm{BT}_{2} \mathrm{~T}_{4}$ oligomer is facing down, whereas in (b) it is $(S)$-oligo$\mathrm{BT}_{2} \mathrm{~T}_{4}$ which constitutes the bottom layer. The induced bending is analog to what has been observed in Figure 3A, but based on different diasteromeric couples, as it is possible to change the chiral configuration of either the probe or of the selector to achieve the same result. In other words, the chiral information can be propagated to the macroscopic scale in two different ways. In the first case (Figure 3A) the chiral recognition occurs between the two molecular antipodes of the probe (D-DOPA and L-DOPA) and one given configuration of the selector. In the second case (Figure 3B) two different selector configurations interact with one and the same enantiomer (DDOPA). Both situations lead to opposite bending due to the diasteromeric interactions between probe and selector.

Since the deformation of PPy is reversible and the composite objects are constituted by a $(R)$ - and $(S)$-oligo-B $\mathrm{B}_{2} \mathrm{~T}_{4}$ face, the curling of the same object can be tuned in different directions by changing the configuration of the chiral probe present in solution.

A more quantitative analysis of Figure $3 \mathrm{~A}$, in terms of the change in bending angle and curvature as a function of time, reveals a fast and continuous increase of both figures of merit. In the presence of D-DOPA, which is selectively transformed at the $(R)-\mathrm{BT}_{2} \mathrm{~T}_{4}$ modified side, facing downwards, the bending angle (Figure S3) and the curvature (Figure S4) start to change almost immediately after switching on the potential because the hybrid layer can freely bend upwards. In contrast, when L-DOPA is in the solution, a short delay can be observed before both parameters start to increase. This is due to the fact that in this case the bending is downwards and therefore the whole object has to be lifted against gravity by the extremity which is bending, and this obviously requires more driving force.

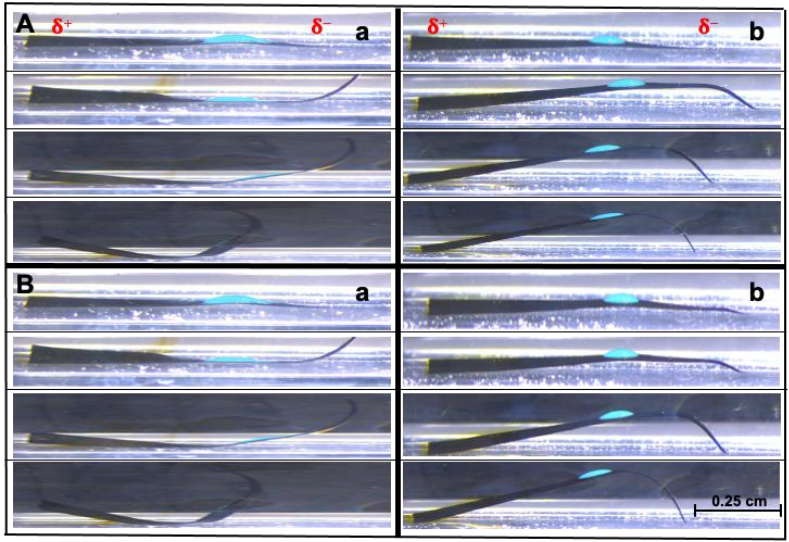

Figure 3. Side view of the triangular hybrid polymer objects, when an electric field of $0.6 \mathrm{~V} \mathrm{~cm}^{-1}$ is applied. A sequence of four time laps images, extracted from Supporting Video S3, are shown for each panel. A) The $(R)-\mathrm{BT}_{2} \mathrm{~T}_{4}$ oligomer is orientated towards the bottom and the object is exposed to a $0.1 \mathrm{M} \mathrm{LiClO}_{4}$ solution containing either (a) D-DOPA or (b) L-DOPA. B) The $(R)-\mathrm{BT}_{2} \mathrm{~T}_{4}$ oligomer is orientated towards either (a) the bottom or (b) the top and the object is exposed to a $0.1 \mathrm{M} \mathrm{LiClO}_{4}$ containing in both cases $5 \mathrm{mM}$ D-DOPA.

At the end of the bipolar experiments, when the curling is completed, the freestanding polymer films can be removed from the solution and dried. The resulting objects are shown in Figure 4. The polymer film illustrated in Figure 4A is the result of the curling experiment depicted in Figure 2A, whereas Figure $4 \mathrm{~B}$ shows the final configuration of the object from Figure $2 \mathrm{~B}$. In the first case $(R)$-oligo- $\mathrm{BT}_{2} \mathrm{~T}_{4}$ was located at the front side of the triangle, whereas in the other case it was at the backside. Therefore, the bending occurs in opposite directions. Exactly the same final results are obtained when depositing $(R)$-oligo- $\mathrm{BT}_{2} \mathrm{~T}_{4}$ always at the same face of the triangle, but exposing the hybrid film either to L- or D-DOPA. Due to the different diastereomeric interactions, the final two objects are specular enantiomorphs, endowed with inherent chirality. This means that molecular chiral information has been successfully transferred to the macroscopic scale in a straightforward way.

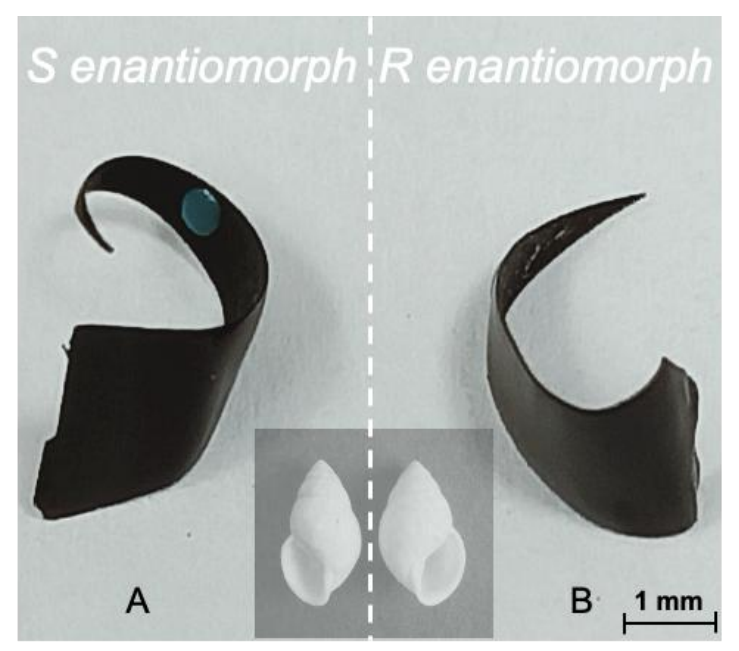

Figure 4. Picture of the two $(R)$ - and $(S)$-enantiomorphs recovered at the end of the bipolar experiments illustrated in Figure 2, 
illustrating the transmission of molecular chirality to the macroscopic scale (scale bar $1 \mathrm{~mm}$ ).

\section{CONCLUSION}

We have confirmed the robustness of the concept of inherent chirality that allows conferring intrinsic chiral features to the materials used as building blocks of hybrid bilayer objects. Following this concept, it is possible to propagate chiral information along different length scales. After starting from the molecular level with the inherently chiral monomers, the next step encompasses the supramolecular scale, via a transmission into helical macromolecular structures during electrooligomerization, and culminates in macroscopic chirality represented by the controlled generation of enantiomorphs through chirospecific curling, triggered by bipolar electrochemistry. Such an artificial version of macroscopic enantiogenesis, based on the synergy of well-chosen molecular ingredients and physico-chemical engineering, can be considered as a model system for the transmission of chiral information through space in natural systems.

\section{MATERIALS AND METHODS}

Synthesis of the polypyrrole films. Pyrrole monomer $(0.2 \mathrm{M})$ was dissolved in Milli Q water with dodecylbenzene sulfonate $(0.25 \mathrm{M})$. After complete dissolution of both components, two gold coated glass slides were positioned parallel in a beaker. The beaker was filled with $12 \mathrm{~cm}^{3}$ of this solution and one gold coated glass slide was used as a working electrode while the other one as counter electrode and $\mathrm{Ag} / \mathrm{AgCl}(3 \mathrm{M} \mathrm{KCl})$ was the reference electrode. A fixed current of 4 $\mathrm{mA}$ was applied for $1.5 \mathrm{~h}$ for the polymerization of pyrrole. After polymerization, the polymer coated substrate was washed with water, dried and used for further oligomerization of 2,2'-bis[2-(5,2'bithienyl)]-3,3'-bithianaphthene chiral monomer.

Electrosynthesis of enantiopure oligo- $(R)$ - or oligo-(S)-2,2'bis[2-(5,2'-bithienyl)]-3,3'-bithianaphthene (oligo- $(R)-$ or $(S)$ $\left.\mathbf{B T}_{2} \mathbf{T}_{4}\right)$. The electrosynthesis of enantiopure oligo- $(S)-\mathrm{BT}_{2} \mathrm{~T}_{4}$ and oligo- $(R)-\mathrm{BT}_{2} \mathrm{~T}_{4}$ was carried out by employing the polypyrrole coated substrate as working electrode in a small beaker containing $10 \mathrm{~cm}^{3}$ of $0.1 \mathrm{M}$ solution of lithium perchlorate $\left(\mathrm{LiClO}_{4}\right)$ in acetonitrile $(\mathrm{MeCN})$ and the $(R)$ - or $(S)$ - enantiopure monomers at $5 \mathrm{mM}$ concentration. The counter electrode was a platinum grid together with an $\mathrm{Ag} / \mathrm{AgCl}$ reference electrode. Oligo- $(S)-\mathrm{BT}_{2} \mathrm{~T}_{4}$ and oligo- $(R)-\mathrm{BT}_{2} \mathrm{~T}_{4}$ were synthetized by chronopotentiometry at a fixed current value of $4 \mathrm{~mA}$ for 40 minutes onto the whole rough polypyrrole surface. After deposition of the oligo-(3,3'dibenzothiophene)-polypyrrole hybrid films, they were peeled off from the gold electrode and then properly cut in a triangular shape, with a $90^{\circ}$ angle. In this way two bilayer systems were obtained: i) one scalene triangle with $(R)$-oligo-B $\mathrm{BT}_{2} \mathrm{~T}_{4}$ on top of the Ppy and ii) one scalene triangle with $(S)$-oligo-BT $\mathrm{B}_{4}$ on top of the Ppy. The two triangles were combined using expoxy glue with the smooth Ppy sides (the ones without the oligomers) facing each other. The resulting hybrid sandwich (see Scheme 1) was used as a bipolar electrode.

SEM analysis of the hybrid sandwich electrode. SEM experiments were carried out using a Hitachi TM-1000 tabletop microscope. SEM micrographs of the cross section of the sandwich-type structures are provided in the SI (Figure S1 and S2).

Differential pulse voltammetry (DPV) experiments. DPV experiments were carried out in a beaker, used as electrochemical cell, containing the enantiomers of L- or D-DOPA $(5 \mathrm{mM})$ dissolved in water and $0.1 \mathrm{M} \mathrm{LiClO}_{4}$. The reference electrode was $\mathrm{Ag} / \mathrm{AgCl}$ and a platinum grid the counter electrode. The working electrodes were hybrid electrodes composed of an oligo- $(S)-\mathrm{BT}_{2} \mathrm{~T}_{4}$ layer or an oligo$(R)-\mathrm{BT}_{2} \mathrm{~T}_{4}$ layer, deposited on a freestanding Ppy film. This film was carefully isolated on the backside with varnish and connected with copper tape to the potentiostat. The optimized DPV parameters used for recording the voltammetric signals of L- or D-DOPA were: step potential $10 \mathrm{mV}$, modulation amplitude $60 \mathrm{mV}$, modulation time 40 ms and interval time $200 \mathrm{~ms}$.

Bipolar curling experiments. For bipolar curling experiments, enantiopure $(R)$ - or $(S)$-oligo-(3,3'-dibenzothiophene)-polypyrrole hybrid triangles were placed in the center of the bipolar cell without any support. Two graphite feeder electrodes were positioned at the extremities of the cell $\left(5 \mathrm{~cm}\right.$ distance). $0.1 \mathrm{M} \mathrm{LiClO}_{4}$ was used as supporting electrolyte to provide a sufficient amount of ions for charge compensation in the conducting polymer during bipolar actuation in the presence of $5 \mathrm{mM} \mathrm{L}$ - or D-DOPA.

The chirality controlled deformation was recorded using a macroscope (LEICA Z16 APO) in video mode.

Movie data treatment was carried out with the help of image $\mathrm{J}$ software.

\section{ASSOCIATED CONTENT}

\section{Supporting Information}

The Supporting Information is available free of charge on the ACS Publications website.

-Video S1 Hybrid lamellar electrode strip exposed to an aqueous solution of $5 \mathrm{mM}$ D-DOPA and $0.1 \mathrm{M} \mathrm{LiCO}_{4},(R)$ - oligo-BT $\mathrm{T}_{4}$ is deposited at the back (.avi)

-Video S2 Hybrid lamellar electrode strip exposed to an aqueous solution of $5 \mathrm{mM}$ D-DOPA and $0.1 \mathrm{M} \mathrm{LiCO}_{4},(R)$ - oligo- $\mathrm{BT}_{2} \mathrm{~T}_{4}$ is deposited at the front (.avi)

-Video S3 Hybrid lamellar electrode strips with $(R)-\mathrm{BT}_{2} \mathrm{~T}_{4}$ oligomer at the bottom exposed to an aqueous solution of either $5 \mathrm{mM}$ D-DOPA or 5mM L-DOPA (.avi)

-SEM characterization of the hybrid layer composition

\section{AUTHOR INFORMATION}

\section{Corresponding Author}

* Alexander Kuhn- Univ. Bordeaux, CNRS UMR 5255, Bordeaux INP, ENSCBP, 16 avenue Pey Berland, 33607 Pessac, France, e-mail: kuhn@enscbp.fr

\section{Present Addresses}

Serena Arnaboldi- Univ. Bordeaux, CNRS UMR 5255 , Bordeaux INP, ENSCBP, 16 avenue Pey Berland, 33607 Pessac, France, Univ. degli Studi di Milano, Dip. di Chimica, Via Golgi 19, 20133 Milano; Italy

Bhavana Gupta- Univ. Bordeaux, CNRS UMR 5255, Bordeaux INP, ENSCBP, 16 avenue Pey Berland, 33607 Pessac, France

Tiziana Benincori- Univ.degli Studi dell'Insubria, Dip. di Scienza e Alta Tecnologia,Via Valleggio 11, 22100 Como, Italy

Giorgia Bonetti- Univ.degli Studi dell'Insubria, Dip. di Scienza e Alta Tecnologia,Via Valleggio 11, 22100 Como, Italy

Roberto Cirilli- Centro Nazionale per il Controllo e la Valutazione dei Farmaci, Istituto Superiore di Sanità, Viale Regina Elena 299, 00161 Roma, Italy

\section{Author Contributions}

S.A. and B.G. designed and performed the experiments; wrote and edited the manuscript. A.K. proposed the research project, provided resources, designed experiments and edited the manuscript. T.B. designed the inherently chiral monomers, G.B. synthetized the inherently chiral monomers, R.C separated the enantiomers of the inherently chiral monomers by chiral HPLC. 


\section{Notes}

The authors declare no competing financial interest.

\section{ACKNOWLEDGMENTS}

The work has been funded by the European Research Council (ERC) under the European Union's Horizon 2020 research and innovation program (grant agreement $n^{\circ} 741251$, ERC Advanced grant ELECTRA). This study has also received financial support from the French State in the framework of the "Investments for the future" program, IdEx Bordeaux (reference ANR-10-IDEX03-02). S.A. gratefully acknowledges the financial support of Università degli Studi di Milano for a post-doc scholarship. The authors are very grateful for fruitful discussions with Patrizia Mussini and Mirko Magni about this project and to Gerardo Salinas for the SEM analysis.

\section{ABBREVIATIONS}

$(R)$ - or $(S)$-2,2'-bis[2-(5,2'-bithienyl)]-3,3'-bithianaphthene $((R)$ or $\left.(S)-\mathrm{BT}_{2} \mathrm{~T}_{4}\right)$; differential pulse voltammetry (DPV); L- or D-3,4dihydroxyphenylalanine (L- or D-DOPA); polypyrrole (Ppy); lithium perchlorate $\left(\mathrm{LiClO}_{4}\right)$; potassium chloride $(\mathrm{KCl})$, acetonitrile $(\mathrm{MeCN})$

\section{REFERENCES}

[1] Morrow, S. M.; Bissette, A. J.; Fletcher, S. P. Transmission of Chirality through Space and Across Length Scales. Nat. Nanotechnol. 2017, 10.1038/NNANO.2017.62.

[2] Feringa, B. L.; van Delden, R. A. Absolute Asymmetric Synthesis: the Origin, Control, and Amplification of Chirality. Angew. Chem. Int. Ed. 1999, 38, 3418-3438.

[3] Iski, E. V.; Tierney, H. L.; Jewell, A. D.; Sykes, E. C. H.; Spontaneous Transmission of Chirality through Multiple Length Scales. Chem. Eur. J. 2011, 17, 7205-7212

[4] Castriciano, M. A.; Gentili, D.; Romeo, A.; Cavallini, M.; Monsù Scolaro, L.; Spatial Control of Chirality in Supramolecular Aggregates. Scientific Reports. 2017, 7:44094

[5] Berthod, A.; Chiral Recognition Mechanisms. Anal. Chem. 2006, 78, 2093-2099.

[6] Fasel, R.; Parschau, M.; Ernst, K. H.; Amplification of Chirality in Two-Dimensional Enantiomorphous Lattices. Nature. 2006, 439, 449-452.

[7] Zhang, W.; Banerjee-Ghosh, K.; Tassinari, F.; Naaman R.; Enhanced Electrochemical Water Splitting with Chiral Molecule-Coated $\mathrm{Fe}_{3} \mathrm{O}_{4}$ Nanoparticles. ACS Energy Lett. 2018, 3, 2308-2313.

[8] O’Neill, M.; Kelly, S. M.; Liquid Crystals for Charge Transport, Luminescence, and Photonics. Adv. Mater. 2003, 15, 1135 1146.

[9] Zheludev, N. I.; The Road Ahead for Metamaterials. Science. 2010, 328, 582-583.

[10] Taylor, M. S.; Jacobsen, E. N.; Asymmetric Catalysis by Chiral Hydrogen-Bond Donors. Angew. Chem. Int. Ed. 2006, 45, 15201543.

[11] Pu, L.; Fluorescence of Organic Molecules in Chiral Recognition. Chem. Rev. 2004, 104, 1687-1716.

[12] Maier, N. M.; Franco, P.; Lindner, W.; Separation of Enantiomers: Needs, Challenges, Perspectives. J. Chromatogr. A. 2001, 906, 3-33.

[13] Heinze, J.; Frontana-Uribe, B. A.; Ludwigs, S.; Electrochemistry of Conducting Polymers-Persistent Models and New Concepts. Chem. Rev. 2010, 110, 4724-4771.

[14] Mukhopadhyay, P.; Zuber, G.; Wipf, P.; Beratan, D. N.; Contribution of a Solute's Chiral Solvent Imprint to Optical Rotation. Angew. Chemie Int. Ed. 2007, 46, 6450-6452.
[15] Mukhopadhyay, P.; Zuber, Goldsmith, M-R.; Wipf, P.; Beratan, D. N.; Solvent Effect on Optical Rotation: A Case Study of Methyloxirane in Water, ChemPhysChem, 2006, 7, 2483-2486.

[16] Kane-Maguire, L.A.P.; Wallace G.G.; Chiral Conducting Polymers. Chem. Soc. Rev. 2010, 39, 2545-2576.

[17] Mondal, P.C.; Fontanesi, C.; Waldeck, D.H.; Naaman R. Spindependent Transport through Chiral Molecules Studied by Spindependent Electrochemistry, Acc. Chem. Res. 2016, 449, 2560-2568.

[18] Arnaboldi, S.; Grecchi, S.; Magni, M.; Mussini, P. Electroactive Chiral Oligo- and Polymer Layers for Electrochemical Enantiorecognition. Curr. Opin. Electrochem. 2018, 7, 188-199.

[19] Lemaire, M.; Delabouglise, D.; Garreau, R.; Guy, A.; Roncali , J.; Enantioselective Chiral Poly(thiophenes). J. Chem. Soc. Chem. Commun. 1988, 658-661.

[20] Dong, L.; Lu, B.; Duan, X.; Xu, J.; Hu, D.; Zhang, K.; Zhu, X.; Sun, H.; Ming, S.; Wang, Z.; Zhen, S. Novel Chiral PEDOTs for Selective Recognition of 3,4-dihydroxyphenylalanine Enantiomers: Synthesis and Characterization, J. Polym. Sci. A1. 2015, 53, 22382251 W.;

[21] S. Sharma Piyush, S.; Pietrzyk-Le, A.; D'Souza, F.; Kutner,

Electrochemically Synthesized Polymers in Molecular Imprinting for Chemical Sensing. Anal. Bioanal. Chem. 2012, 402, 3177-3204.

[22] Sannicolò, F.; Rizzo, S.; Benincori, T.; Kutner, W.; Noworyta, K.; Sobczak, J. W.; Bonometti, V.; Falciola, L., Mussini, P. R.; Pierini, M. An Effective Multipurpose Building Block for 3D Electropolymerisation: 2,2'-bis(2,2'-bithiophene-5-yl)-3,3'bithianaphthene. Electrochim. Acta 2010, 55, 8352-8364

[23] Sannicolò, F.; Arnaboldi, S.; Benincori, T.; Bonometti, V.; Cirilli, R.; Dunsch, L.; Kutner, W.; Longhi, G.; Mussini, P.R.; Panigati, M.; Pierini, M.; Rizzo, S. Potential- Driven Chirality Manifestations and Impressive Enantioselectivity by Inherently Chiral Electroactive Organic Films. Angew. Chem. Int. Ed. 2014, 53, 26232627.

[24] Arnaboldi, S.; Benincori, T.; Cirilli, R.; Grecchi, S.; Santagostini, L.; Sannicolò, F.; Mussini P.R. "Inherently Chiral" Thiophene-Based Electrodes at Work: a Screening of Enantioselection Ability toward a Series of Pharmaceutically Relevant Phenolic or Catecholic Amino Acids, Amino Esters, And Amine. Anal. Bioanal. Chem. 2016, 408, 7243-7254.

[25] Arnaboldi, S.; Benincori, T.; Cirilli, R.; Kutner, W.; Magni, M., Mussini, P. R.; Noworyta, K.; Sannicolò, F.; Inherently Chiral Electrodes: the Tool for Chiral Voltammetry. Chem. Sci. 2015,6, 1706-1711.

[26] Grecchi, S.; Arnaboldi, S.; Korb, M.; Cirilli, R.; Araneo, S.; Guglielmi, V.; Tomboni, G.; Magni, M.; Benincori, T.; Lang, H.; Mussini, P.R. Widening the Scope of "Inherently Chiral" Electrodes: Enantiodiscrimination of Chiral Electroactive Probes with Planar Stereogenicity. Chem. Electro. Chem. 2020, 7, 3382-3382.

[27] Attard, G. A. Electrochemical Studies of Enantioselectivity at Chiral Metal Surfaces. J. Phys. Chem. B 2001, 105, 3158-3167.

[28] Hazzazi, O. A.; Attard, G. A.; Wells, P.B. Molecular Recognition in Adsorption and Electro-Oxidation at Chiral Platinum Surfaces. 2004, 216, 247-255.

[29] Kelso, M. V.; Tubbesing, J. Z.; Chen, Q.; Switzer, J. A. Epitaxial Electrodeposition of Chiral Metal Surfaces on Silicon(643). $J$. Am. Chem. Soc. 2018, 140, 15812-15819.

[30] Arnaboldi, S.; Gupta, B.; Benincori, T.; Bonetti, G.; Cirilli, R.; Kuhn, A. Absolute Chiral Recognition with Hybrid Wireless Electrochemical Actuators. Anal. Chem., 2020, 92, 10042-10047.

[31] Assavapanumat, S.; Gupta, B.; Salinas, G.; Goudeau, B.; Wattanakit, C.; Kuhn, A. Chiral Platinum-polypyrrole Hybrid Films as Efficient Enantioselective Actuators. Chem. Commun. 2019, 55, 10956-10959.

[32] Zhang, L.; Gupta, B.; Goudeau, B.; Mano, N.; Kuhn, A. Wireless Electromechanical Readout of Chemical Information. J. Am. Chem. Soc. 2018, 140, 15501-15506.

[33] Pyo, M.; Bohn, C. C.; Smela, E.; Reynolds, J. R.; Brennan, A. B. Direct Strain Measurement of Polypyrrole Actuators Controlled by the Polymer/Gold Interface. Chem. Mater. 2003, 15, 916-922. 
[34] Gao, J.; Sansiñena, J.-M.; Wang, H.-L. Tunable Polyaniline Chemical Actuators. Chem. Mater. 2003, 15, 2411-2418.

[35] Gupta, B.; Afonso, M. C.; Zhang, L.; Ayela, C.; Garrigue, P.; Goudeau, B.; Kuhn, A. Wireless Coupling of Conducting Polymer Actuators with Light Emission. ChemPhysChem 2019, 20, 941-945.

[36] Gupta, B.; Goudeau, B.; Kuhn, A. Wireless Electrochemical Actuation of Conducting Polymers. Angew. Chem. Int. Ed. 2017, 56, 14183-14186.
[37] Gupta, B.; Goudeau, B.; Garrigue, P.; Kuhn, A. Bipolar Conducting Polymer Crawlers Based on Triple Symmetry Breaking. Adv. Funct. Mater. 2017, 28, 1705825. 


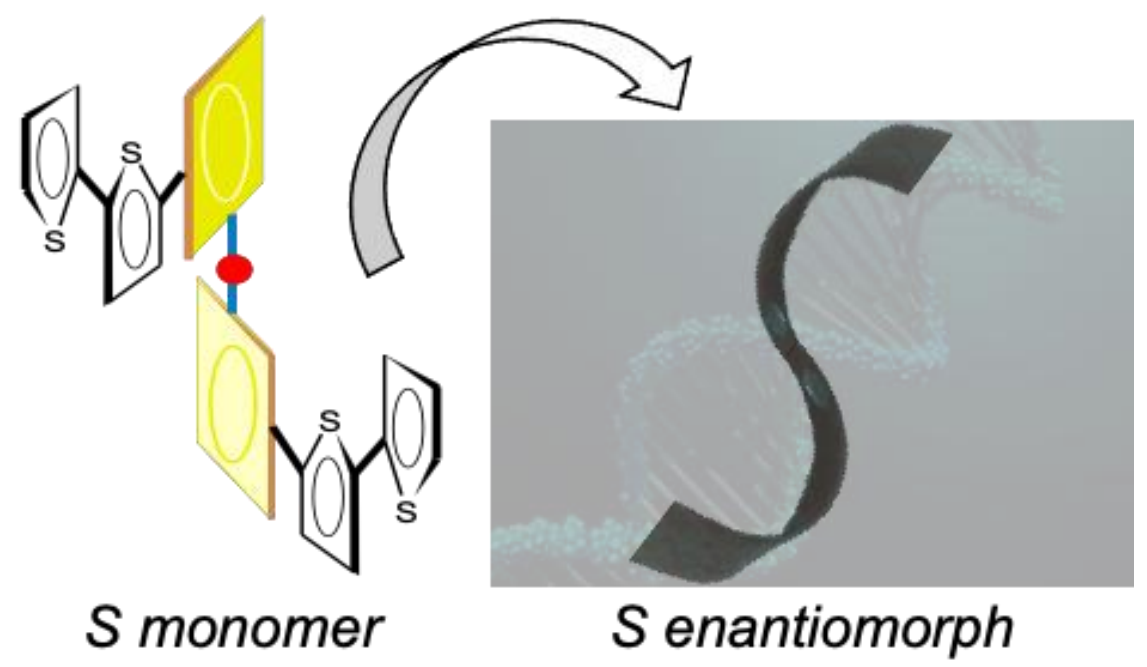

